\title{
Intrapopulation Antagonism Can Reduce the Growth and Aggressiveness of the Wheat Head Blight Pathogen Fusarium graminearum
}

\author{
Martha M. Vaughan, † Todd J. Ward, Susan P. McCormick, Nathane Orwig, William T. Hay, Robert Proctor, and \\ Debra Palmquist \\ U.S. Department of Agriculture, Agricultural Research Services, National Center of Agricultural Utilization Research, Mycotoxin Prevention \\ and Applied Microbiology Unit, Peoria, IL 61604 \\ Accepted for publication 27 January 2020.
}

\begin{abstract}
Fusarium graminearum is a causal agent of Fusarium head blight (FHB), a disease that reduces yield and quality of cereal crops and contaminates grain with mycotoxins that pose health risks to humans and livestock. Interpopulation antagonistic interactions between isolates that produce different trichothecene mycotoxins can reduce FHB in wheat, but it is not known if interactions between isolates with a shared population identity that produce the same trichothecenes have a similar effect. Using isolates from the predominant $F$. graminearum populations in North America (NA1 and NA2), we examined intrapopulation interactions by comparing growth, disease progression, and toxin production of individual isolates with multi-isolate mixes. In vitro, mycelial growth was significantly greater when most NA1 and NA2 isolates were cultured individually versus when cultured as a mixture of isolates from the same population. In susceptible wheat Norm, FHB generally progressed faster in heads inoculated with an individual isolate versus a multi-isolate
\end{abstract}

ABSTRACT mixture, but the antagonistic effect of intrapopulation interactions was more pronounced for NA1 than NA2 isolates. By contrast, in moderately resistant wheat Alsen, mixtures of isolates from either population caused obvious reductions in FHB development. Mycotoxin contamination was not consistently affected by intrapopulation interactions and varied depending on the interacting isolates from either population. Our results indicate that antagonistic intrapopulation interactions can influence FHB in controlled environmental conditions. Understanding if the regional composition of pathogen populations similarly influences FHB in the field could improve disease forecasting and management practices.

Keywords: antagonism, disease control and pest management, ecology and epidemiology, Fusarium graminearum, Fusarium head blight, intrapopulation interactions, mycotoxins, pathogen aggressiveness, population biology, wheat
In many regions of the world, the fungus Fusarium graminearum is the predominant cause of Fusarium head blight (FHB), a devastating disease that affects small cereal grains such as wheat, barley, and oats. Over the past few decades, the fungus has caused widespread outbreaks of FHB and multibillion dollar losses to the wheat industry in North America (Nganje et al. 2004). FHB can reduce crop yield and contaminate grain with trichothecene mycotoxins, such as deoxynivalenol (DON), that threaten food and feed safety (McMullen et al. 2012). Trichothecenes impair protein synthesis in eukaryotic cells, and can cause nausea, vomiting, anorexia, and adversely affect digestive, immune, and neurological function (Pestka 2010; Wu et al. 2014). Additionally, in wheat, DON functions as a virulence factor allowing the pathogen to overcome plant defenses and spread beyond the spikelet it initially infects (Bai et al. 2002; Mesterhazy et al. 1999).

In the United States and Canada, two genetically distinct populations of $F$. graminearum, termed NA1 and NA2, are responsible for the majority of FHB (Gale et al. 2007; Kelly et al.

†Corresponding author: M. M. Vaughan; martha.vaughan@usda.gov

Mention of trade names or commercial products in this article is solely for the purpose of providing specific information and does not imply recommendation or endorsement by the U.S. Department of Agriculture (USDA). USDA is an equal opportunity provider and employer.

*The $\boldsymbol{e}$-Xtra logo stands for "electronic extra" and indicates that supplementary tables are published online.

The author(s) declare no conflict of interest.

This article is in the public domain and not copyrightable. It may be freely reprinted with customary crediting of the source. The American Phytopathological Society, 2020.
2015; Liang et al. 2015; Ward et al. 2008). Different DON production phenotypes (chemotypes) have been recognized among isolates, including the 3-acetyldeoxynivalenol (3ADON) chemotype and the 15-acetyldeoxynivalenol (15ADON) chemotype (Miller et al. 1991). Isolates belonging to the endemic NA1 population typically produce $15 \mathrm{ADON}$, are widespread in the United States and Canada, and are genetically diverse. In contrast, NA2 isolates typically produce $3 \mathrm{ADON}$ and are less genetically diverse than NA1 isolates, likely due to a genetic bottleneck associated with a relatively recent introduction into North America (Kelly and Ward 2018; Ward et al. 2008). While these chemotypes are distinct in vitro, in planta both $3 \mathrm{ADON}$ and $15 \mathrm{ADON}$ are typically deacetylated to form DON.

NA1 appears to be native to North America and is widely distributed across major wheat growing regions in the United States and Canada. In contrast, NA2 may have been introduced into North America in the last several decades and is sympatric with NA1 in the northern United States and southern Canada (Gale et al. 2007; Ward et al. 2008). Despite evidence of recombination the two populations remain distinct. On average, NA2 isolates grow faster in vitro, are more aggressive, and can contaminate grain with more toxin than NA1 isolates on certain wheat lines (Foroud et al. 2012; Gilbert et al. 2010; Puri and Zhong 2010; Von der Ohe and Miedaner 2011; Ward et al. 2008). Perhaps as a result of these differences, NA2 isolates appear to have an adaptive advantage over NA1 isolates in some environments. The frequency of NA2 isolates has rapidly increased in western and Maritime provinces of Canada, and in parts of the Upper Midwest of the United States (Kelly et al. 2015; Liang et al. 2015; Ward et al. 2008). However, the frequency of NA2 isolates does not appear to have increased in the eastern Canadian provinces of Quebec and Ontario, indicating that the two populations are responding differently in a manner consistent with a complex adaptive landscape (Kelly et al. 2015). 
Multiple diverse Fusarium species and isolates of $F$. graminearum can coexist within a field during the growing season (Kelly et al. 2015; Xu et al. 2005), on crop residues, and in the soil (Goswami and Kistler 2004). Interspecies competitive interactions between FHB pathogens have been reported (Wagacha et al. 2012). For example, coinoculations of wheat with $F$. graminearum and $F$. culmorum resulted in competitive interactions that altered the reisolation rate of each species in a manner that was dependent on environmental factors (Miedaner et al. 2004; Siou et al. 2015b; Von der Ohe and Miedaner 2011; Xu et al. 2007). Intraspecies competitive interactions between $F$. graminearum isolates have also been demonstrated (Von der Ohe and Miedaner 2011; Walkowiak et al. 2015). Wheat heads coinoculated with an $F$. graminearum $3 \mathrm{ADON}$ producing isolate and an $F$. graminearum $15 \mathrm{ADON}$ producing isolate resulted in less FHB symptoms and DON contamination compared with heads infected with a single isolate (Walkowiak et al. 2015). However, it is unclear if intraspecies interactions between isolates with the same population identity and trichothecene chemotype affect pathogen growth, FHB development, and DON contamination in wheat.

Since individual $F$. graminearum isolates of the same population and chemotype also likely compete for resources within coinfected wheat heads, we hypothesized that intrapopulation antagonism would influence growth rate in vitro and FHB development and DON contamination in wheat. We further hypothesized that the degree of this antagonism would be dependent on the genetic diversity of the population. To test these hypotheses, we assessed in vitro mycelial growth, and in planta FHB development and DON contamination resulting from four isolates alone compared with an intrapopulation mixture of the four isolates. These experiments were conducted with four representative NA1 isolates that produce $15 \mathrm{ADON}$, and four NA2 isolates that produce 3ADON. Two geographically distinct sets of isolates for each population were used to assess if in planta results were specific to a particular set of isolates. Additionally, all FHB assays with individual isolates and their corresponding mixes were performed in a susceptible and in a moderately resistant spring wheat cultivar.

\section{MATERIALS AND METHODS}

F. graminearum isolates. A total of 16 isolates were selected from previously published collections of $F$. graminearum recovered from wheat heads in the upper Midwestern United States and southern Canada (Table 1). Isolates were selected to represent the natural variation within four genetic/geographic populations (NA1 and NA2 populations from the Midwestern United States and NA1 and NA2 populations from southern Canada). Isolate chemotypes were determined using multilocus genotyping or gas chromatography-mass spectrophotometry (GC-MS), and high confidence population assignments (probability of membership $\geq 99 \%$ ) were inferred from analyses of restriction fragment length polymorphism or variable number tandem repeat genotypes (Gale et al. 2007; Kelly et al. 2015; Liang et al. 2015; Ward et al. 2008).

Experimental design. To assess the effect of intrapopulation interactions, we compared in vitro mycelial growth, FHB development, and toxin production of four individual isolates to a multiisolate mixture of the four isolates. Treatments for each genetic population consisted of four representative isolates and a mixture of these isolates at equal proportions (mix). Combinations of four isolates (versus pairs of isolates) were chosen to increase the diversity of the interacting isolates and capture a more representative sample of the natural population variation. In vitro growth assays were performed in triplicate with only the Midwestern U.S. isolates (Table 1). For the wheat head blight assays, three experimental replicates were performed with the Midwestern United States, and one was performed with an alternate set of isolates from southern Canada. Whenever possible, NA1 and NA2 experimental replicates were conducted at the same time so that intra- and interpopulation comparisons could be made. However, due to growth chamber space limitations and the need to maximize treatment head number, this was not always possible.

Prior to experimental testing, strains were reisolated from a single conidia and grown for 7 days at $24^{\circ} \mathrm{C}$ on V8 plates. Several agar plugs were harvested from each of these plates and placed in a $60 \%$ glycerol stock solution for storage at $-80^{\circ} \mathrm{C}$. For each experimental replication, single isolates were recovered from glycerol stocks by placing two agar plugs in $10 \mathrm{ml}$ of mung bean broth. Cultures were then grown at $28^{\circ} \mathrm{C}$ for $48 \mathrm{~h}$ with shaking (200 rpm) (Gale et al. 2007). The conidial suspension of each single isolate culture was concentrated via centrifugation and then diluted to $10^{5} \mathrm{ml}^{-1}$ in mung bean broth, based on cell counts obtained using a Scepter automated cell counter (Millipore, Billerica, MA). Multi-isolate cultures for each population were prepared by adding equal volumes of the four single isolate cultures which had been adjusted to $10^{5} \mathrm{ml}^{-1}$ conidia. Fresh inoculum was prepared on the day of each experiment.

In vitro growth assay. To evaluate growth differences between individual isolate cultures and multi-isolate cultures, we used a modified 96-well microtiter-based method for fungal growth (Baldwin et al. 2019; Mogg et al. 2019), which measured optical density (OD) in liquid media at $600 \mathrm{~nm}$ wavelength over a $48 \mathrm{~h}$ time period. Mung bean cultures described above were adjusted to $5 \times$ $10^{4}$ cells $/ \mathrm{ml}$ and then diluted 1:1 with carboxymethylcellulose medium, and $200 \mu \mathrm{l}$ of the resulting solution was placed into a single well of a Costar 96-well plate (Corning 3595). Each plate contained eight wells (biological replicates) for each of the 10 treatments (four-single NA1 isolates, one- NA1 mix, four-single NA2 isolates, one-NA2 mix) and the inoculated medium control. Prior to reading, plates were incubated for $14 \mathrm{~h}$ at $27^{\circ} \mathrm{C}$ with $12 \mathrm{~h} \mathrm{UV}$ light-dark cycles to stimulate conidial growth. Plates were then moved to a Bioteck Synergy HT plate reader set at $27^{\circ} \mathrm{C}$, and OD was measured every $2 \mathrm{~h}$. Three independent experimental replicates were performed.

TABLE 1. Description of Fusarium graminearum isolates used in this study

\begin{tabular}{|c|c|c|c|c|}
\hline $\mathrm{ID}^{\mathrm{a}}$ & Population & Chemotype & $\begin{array}{l}\text { Geographic } \\
\text { location }\end{array}$ & Reference \\
\hline $06-270$ & NA1 & $15 \mathrm{ADON}$ & $\begin{array}{l}\text { South Dakota, } \\
\text { U.S.A. }\end{array}$ & Liang et al. 2015 \\
\hline $06-225$ & NA1 & $15 \mathrm{ADON}$ & $\begin{array}{l}\text { North Dakota, } \\
\text { U.S.A. }\end{array}$ & Liang et al. 2015 \\
\hline $06-219$ & NA1 & $15 \mathrm{ADON}$ & $\begin{array}{l}\text { North Dakota, } \\
\text { U.S.A. }\end{array}$ & Liang et al. 2015 \\
\hline 13MN1-6 & NA1 & $15 \mathrm{ADON}$ & $\begin{array}{l}\text { Minnesota, } \\
\text { U.S.A. }\end{array}$ & Liang et al. 2015 \\
\hline $00-588$ & NA2 & 3ADON & $\begin{array}{l}\text { Minnesota, } \\
\text { U.S.A. }\end{array}$ & Gale et al. 2007 \\
\hline 12SD6-2 & NA2 & $3 \mathrm{ADON}$ & $\begin{array}{l}\text { South Dakota, } \\
\text { U.S.A. }\end{array}$ & Liang et al. 2015 \\
\hline $06-239$ & NA2 & $3 \mathrm{ADON}$ & $\begin{array}{l}\text { North Dakota, } \\
\text { U.S.A. }\end{array}$ & Liang et al. 2015 \\
\hline $06-240$ & NA2 & $3 \mathrm{ADON}$ & $\begin{array}{l}\text { North Dakota, } \\
\text { U.S.A. }\end{array}$ & Liang et al. 2015 \\
\hline \multicolumn{5}{|l|}{ NRRL } \\
\hline $\begin{array}{l}37408 \\
\text { NRRL }\end{array}$ & \multicolumn{3}{|c|}{ NRRL } & Ward et al. 2008 \\
\hline \multicolumn{5}{|l|}{ NRRL } \\
\hline $\begin{array}{l}37459 \\
\text { NRRL }\end{array}$ & NA1 & $15 \mathrm{ADON}$ & $\begin{array}{l}\text { Manitoba, Canada } \\
\text { Saskatchewan. }\end{array}$ & Ward et al. 2008 \\
\hline 37497 & NA1 & $15 \mathrm{ADON}$ & $\begin{array}{l}\text { Saskatcnewan, } \\
\text { Canada }\end{array}$ & Ward et al. 2008 \\
\hline NRRL & & & & \\
\hline $\begin{array}{l}37525 \\
\text { NRRL }\end{array}$ & NA2 & $3 \mathrm{ADON}$ & Manitoba, Canada & Ward et al. 2008 \\
\hline 52008 & NA2 & $3 \mathrm{ADON}$ & Manitoba, Canada & Kelly et al. 2015 \\
\hline $\begin{array}{l}\text { NRRL } \\
52195\end{array}$ & NA2 & 3ADON & $\begin{array}{l}\text { Saskatchewan, } \\
\text { Canada }\end{array}$ & Kelly et al. 2015 \\
\hline $\begin{array}{l}\text { NRRL } \\
52429\end{array}$ & NA2 & 3ADON & Alberta & Kelly et \\
\hline
\end{tabular}

${ }^{a}$ Strains designated by NRRL were obtained from the Northern Regional Research Laboratory culture collection. 
Plant growth and wheat head blight assays. FHB disease progression assays were performed using susceptible and moderately resistant hard red spring wheat cultivars Norm and Alsen, respectively. Five plants of each cultivar were grown in each 2.5liter plastic pot containing propagation mix (Sun Gro Horticulture, Agawam, MA). Two weeks after germination and then every other week thereafter the pots were fertilized with $500 \mathrm{ml}$ of solution containing 0.5 g liter $^{-1}$ of Peters 20-20-20 (Grace-Sierra Horticultural Products, Milpitas, CA). Three independent experimental replicates (A, B, and $\mathrm{C}$ ) were performed with isolates from the United States, and for comparison, an additional experiment (D) was performed using isolates from Canada. The number of biological replicates per treatment varied across experiments ( 10 to 24 heads per treatment) due to differences in the number of available flowering heads. Wheat plants used in experimental replicate A in the Norm cultivar were initially germinated and grown in a sunlit greenhouse supplemented with high-pressure sodium lights to maintain a $14 \mathrm{~h}$ day cycle. Greenhouse temperatures ranged from 25 to $28^{\circ} \mathrm{C}$ during the day and 17 to $20^{\circ} \mathrm{C}$ at night. At the flag leaf stage, the plants were then transferred to climate-controlled growth chambers maintained at $25^{\circ} \mathrm{C}$ day $/ 20^{\circ} \mathrm{C}$ night, $500 \mathrm{mmol} \mathrm{m} \mathrm{m}^{-2} \mathrm{~s}^{-2}$ photosynthetic photo flux density $14 \mathrm{~h}$ photoperiod and 50 to $60 \%$ relative humidity. However, due to an outbreak of powdery mildew in the greenhouse, plants for all other experimental replicates $(\mathrm{B}, \mathrm{C}$, and $\mathrm{D})$ were grown in the chamber throughout their life cycle.

Flowering heads were inoculated by injecting a single floret with $10 \mu \mathrm{l}$ of $10^{5} \mathrm{ml}^{-1}$ conidia (individual isolates or mix) in mung bean broth, or with sterile mung bean broth as a negative control. Following inoculation, wheat heads were covered with plastic bags for 3 days to ensure high humidity during the initial phase of pathogenesis. Each pot contained only one treatment type, and all the pots were arranged at random within the chamber. The number of diseased florets was counted 1 week after inoculation and then approximately every third day thereafter for 21 days. The cumulative percent of diseased florets was calculated as the percentage of the total number of florets displaying premature whitening or necrosis.

Mycotoxin analyses. Following evaluation of disease progression at 21 days postinoculation (DPI), the inoculated heads were collected for DON analysis. The intact wheat heads were ground, and DON was extracted from 0.8 to $1 \mathrm{~g}$ of the powder with $10 \mathrm{ml}$ of extraction solvent (acetonitrile-water, 86:14, vol/vol) in a $50 \mathrm{ml}$ polypropylene screw-cap centrifuge tube with shaking for $15 \mathrm{~min}$. After centrifugation, a portion of each extract was purified by forcing $5 \mathrm{ml}$ of extract through a MycoSep 225 Trich cartridge (Romer Labs, Union, MO). A 2-ml aliquot of the purified extract were then transferred to a 1 dram vial and concentrated under a nitrogen stream. Trimethylsilyl (TMS) derivatives were prepared by adding $100 \mu \mathrm{l}$ of a 100:1 freshly prepared mixture of N-trimethylsilylimadazole/ trimethylchlorosilane (TMSI/TMCS (Sigma-Aldrich, St. Louis, MO) to the dried extract. After $30 \mathrm{~min}, 900 \mu \mathrm{l}$ of isooctane was added to the reaction mixture followed by $1 \mathrm{ml}$ of water. The mixture was agitated gently until the organic (top) layer became translucent. The organic layer was then transferred to 2-ml autosampler vials for GC-MS analysis. TMS derivatives of purified DON (0.3125 to $80 \mu \mathrm{g})$ were similarly prepared with TMSI/TMCS and used to construct a standard curve for quantification.

GC-MS analyses were performed on an Agilent 7890 gas chromatograph fitted with an HP-5MS column $(30 \mathrm{~m}, 0.25 \mathrm{~mm}$, $0.25 \mu \mathrm{m})$ and a 5977 mass detector. The injection temperature was kept at $250^{\circ} \mathrm{C}$ and the column flow rate was $1 \mathrm{ml} \mathrm{min}-1$. A temperature program was used with initial column temperature of $150^{\circ} \mathrm{C}$ for $1 \mathrm{~min}$, and then increased to 280 at $30^{\circ} \mathrm{C} \mathrm{min}-1$ and held for $3.5 \mathrm{~min}$. Selective ion monitoring (SIM) was applied to detect the characteristic ions of triTMS-DON with fragment ion $(\mathrm{m} / \mathrm{z}$ value) of 235.1 as the target ion and 259.1, 295.1, 392.2, 422.2, and 512.2 as reference ions.

Statistical analyses. Statistical analyses were performed using TableCurve 2D version 5.01 (SYSTAT Software Inc., 2002) and
JMP version 10 (SAS Institute Inc., Cary, NC [2012]). We performed a weighted least squares regression analysis to predict growth rates for each treatment based on OD values measured at $2 \mathrm{~h}$ intervals. A standard weighting $\left(1 / \sigma^{2}\right)$ was used to account for variability across replicate wells in the plate during parameter estimation. To model the growth rate of each culture, weighted regression equations were fit to observed OD values as a function of DPI. Degrees of freedom adjusted $R^{2}$ values were used to select the most parsimonious model that best explained the variance in the data. Initially, overall differences in growth rates between single and multi-isolate treatments were evaluated based on an $F$-test comparing full (including treatment parameters) and reduced (intercept only) models. In addition, differences in OD values were assessed at specific time points by comparing the $95 \%$ confidence intervals for regression predicted OD values of single and multi-isolate cultures. Significant differences were inferred when the $95 \%$ confidence intervals for a single isolate did not overlap the interval of the multi-isolate culture. A separate regression analysis was performed to compare growth rates of NA1 isolates to NA2 isolates. In this analysis, equations were estimated for NA1 and NA2 separately, by comparing OD values from the four single isolate cultures of one population to the other. As before, significant differences between the two populations were inferred based on nonoverlapping $95 \%$ confidence intervals.

Weighted regression equations were also fit to observed mean cumulative percent diseased florets as a function of DPI to predict disease progression rates for all treatments. The most parsimonious models for treatment growth rates were selected as described above. Overall differences in the disease progression rates of single and multi-isolate treatments within a population were evaluated as above using an $F$-test. In addition to comparing disease progression rates, significant differences in disease between single and multiisolate treatments within each population were assessed at each DPI based on nonoverlapping 95\% confidence intervals for regression predicted mean cumulative percent diseased florets, bounded at $100 \%$ disease.

For statistical analyses of toxin data, the DON concentrations were $\log$ transformed to ensure normality, and a Welch's test (Welch 1947) was performed to test for homogeneity of variances across treatments. If no significant difference was found among the treatment variances within an experimental replicate (Welch's test, $P>0.05$ ), a Dunnett's test (Dunnett 1955) was performed to compare the mean DON concentration in heads treated with each single isolate to the mean in heads treated with the multi-isolate mix for each population. However, for experiments where there were significant differences in treatment variances (Welch's test, $P<$ 0.05 ), a nonparametric comparison of DON concentrations between single and multi-isolate treatments was performed using the Steel's test (Steel 1959).

\section{RESULTS}

In vitro growth assays. To assess potential intrapopulation interactions in the absence of the host, individual isolates and multiisolate mixtures were grown in liquid medium, and their growth rates were compared. Software generated regression equations which were used to model culture growth rates based on fit to the observed OD values as a function of DPI are listed in Supplementary Table S1. The predicted growth rate models for all treatments were a good fit to the observed OD values as validated by their $R^{2}$ values, which were greater than 0.99. Regression predicted growth rates for individual and multi-isolate mixed cultures did not significantly differ among the three experimental replicates $(F$-test, $P>0.05)$, indicating that OD values provided consistent estimates of fungal growth across experiments. Therefore, growth data from all three experimental replicates were combined for analysis and visualization (Fig. 1). Full and reduced models analyses determined that each of the individual isolate growth rates significantly differed from the growth rate of the 
corresponding population mix $(P<0.01)$. Furthermore, according to the specific time point assessment of differences, at least one of the individual NA1 isolates had a significantly greater OD value than the NA1 mix at all the time points analyzed (Fig. 1A). At the 8- and 16-h time points, there were few significant differences between the growth rates of individual isolates and the mix, and the individual isolates that were significantly different from the mix varied across these early time points. At the 24-h time point, three of the four individual NA1 isolates had separated and were significantly greater than the NA1 mix. Three of the four individual NA1 isolates were significantly greater at $48 \mathrm{~h}$ as well, but the three isolates with the greater OD values were not consistent across these time points. Isolate 06-225, which initially grew faster and had a higher OD at 16 to $32 \mathrm{~h}$, had significantly lower growth at $48 \mathrm{~h}$ in comparison with the NA1 mixed culture.

At the 48-h time point, all four individual NA2 isolates had significantly greater OD values than the NA2 mix culture (Fig. 1B). However, initially three out of the four isolates grew significantly more slowly than the NA2 mix and these isolates consistently had lower OD values than the mix at 8,16 , and $24 \mathrm{~h}$. It was not until the 32-h time point that three of the four individual NA2 isolates had significantly greater OD values than the NA2 mix culture.

Population-level differences in growth were also detected. Starting at the 16-h time point, the average OD value of the NA2 cultures was significantly greater than the NA1 (Fig. 1C).

Wheat head blight assays. To elucidate the effect of $F$. graminearum intrapopulation interactions on wheat head blight development, wheat heads were point inoculated with individual isolates and multi-isolate mixtures, disease progression was tracked over time, and the rate of disease spread was compared. The software generated regression equations for disease spread rates provided a good fit for the cumulative percent of diseased florets measured over time, as $R^{2}$ values were greater than 0.9 for all treatments in each experiment (Supplementary Tables S2 and S3). In all experimental replicates for both populations and cultivars, significant differences were observed between the rate of disease spread in heads inoculated with individual isolates and the rate of spread in heads inoculated with a multi-isolate mixture $(F$-test, $P<$ $0.05)$, suggesting that intrapopulation interactions within a wheat head influenced head blight progression.

Head blight progression assays in Norm were analyzed individually because the rate of disease progression differed across experimental replicates $(F$-test, $P>0.05$ ) for all isolates except 06 239 (Fig. 2). For most timepoints, significant differences in FHB scores indicated that disease progressed more quickly in heads inoculated with individual isolates as compared with the multiisolate mix suggesting that the intrapopulation interactions were antagonistic to disease progression. Despite experimental variability, the average FHB scores for the individual NA1 strains were greater than that of the average NA1 multi-isolate mix, and at least two of the individual NA1 strains in all three experimental replicates had significantly greater disease than the NA1 mix at two or more timepoints. However, only 06-270 consistently produced significantly more disease than the NA1 mix in all three experiments, although the time points with significant differences varied across replicates. While most of the significant differences detected between individual NA2 isolates and the NA2 mix also suggested that disease progression was greater for inoculations with individual isolates, there was an exception. In experimental replicate $A$, the NA2 isolate 12SD6-2 displayed significantly less disease than the NA2 mix at 14, 17, and 21 DPI (Fig. 2A).

To assess if the observed intrapopulation antagonism was due to the specific isolates utilized, disease assays were also conducted with a set of four Canadian $F$. graminearum isolates (Fig. 2D). Norm wheat heads inoculated with individual Canadian isolates similarly developed disease faster than those inoculated with multi-isolate mixtures, and all of the significant differences indicated that individual Canadian isolate inoculations had produced more
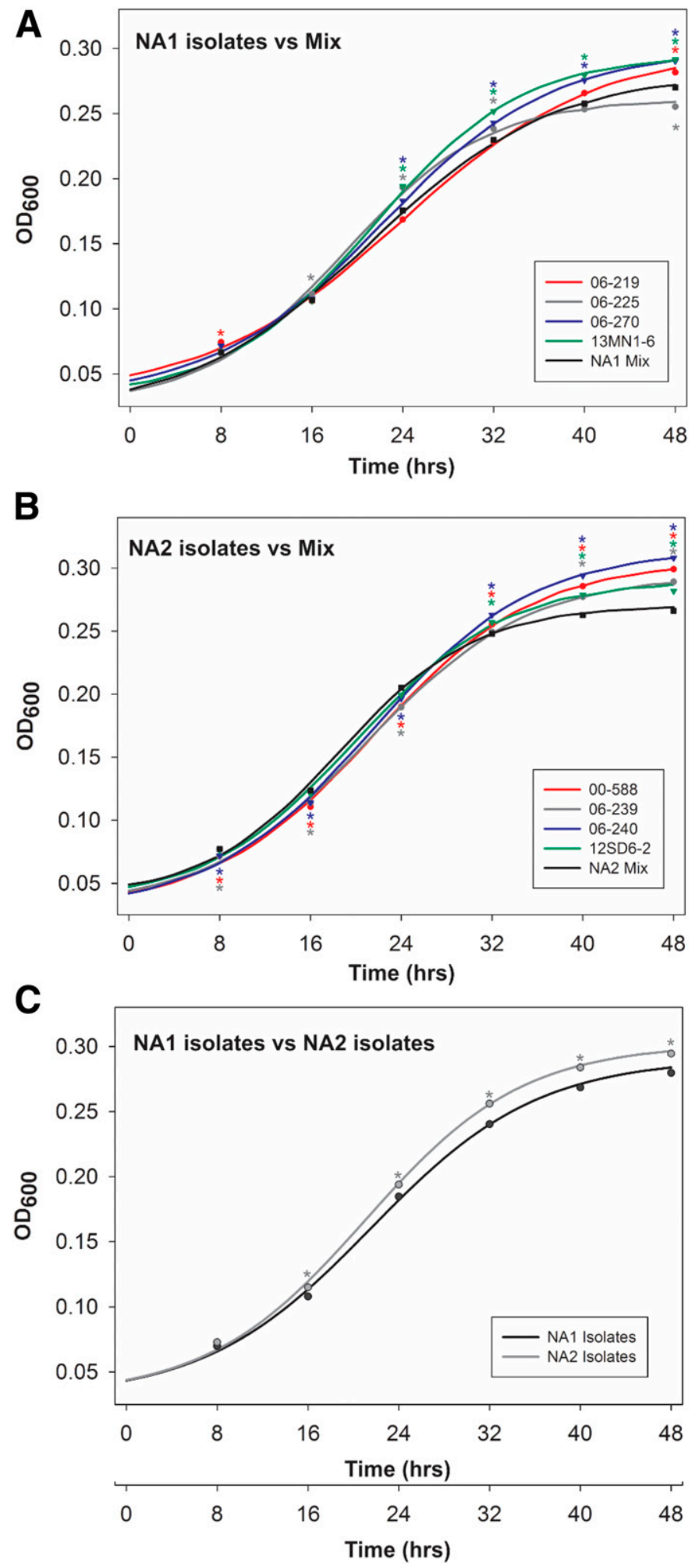

Fig. 1. Intrapopulation interactions affect growth of NA1 or NA2 Fusarium graminearum in liquid culture. Growth rates (solid lines) for individual isolate and multi-isolate cultures (Mix) were predicted using weighted regression of optical density values measured at $600 \mathrm{~nm}$ wavelength $\left(\mathrm{OD}_{600}\right)$ every $2 \mathrm{~h}$ over a $48 \mathrm{~h}$ time period. Measurements were averaged across eight replicate wells and three experimental replicates for each treatment (symbols) and are depicted above at $8 \mathrm{~h}$ intervals. Asterisks indicate significant differences in growth between individual isolates and mixes for A, NA1 or B, NA2, and C, between individual NA1 and NA2 isolates based on nonoverlapping 95\% confidence intervals. Asterisks are color coded by treatment and placed above the regression curves to indicate significantly higher values or below the curves to indicate significantly lower values. Regression equations can be found in Supplementary Table S1. 
disease than the multi-isolate mixtures. Therefore, the observed results were not due to the specific strains selected and appear representative of intrapopulation interactions within NA1 and NA2.

Although both populations appeared to exhibit antagonistic intrapopulation interactions as indicated by reduced disease progression in mixed inoculations, fewer significant differences between single and mixed inoculations were observed in comparisons of the NA2 population. Across all the Norm experimental replicates and time points, 14 significant differences were found between the disease progression of individual NA2 isolates and the NA2 mix. This
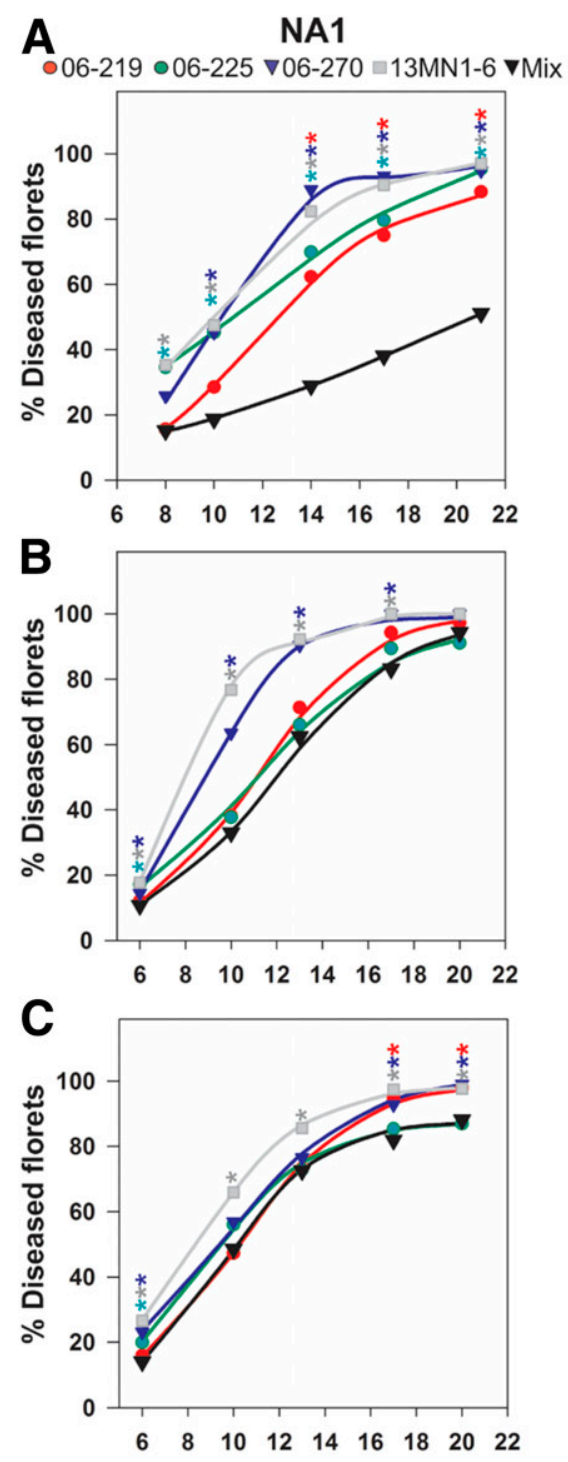

D $\bullet 37408 \bullet 37450 \nabla 37459 \backsim 37497 \nabla$ Mix

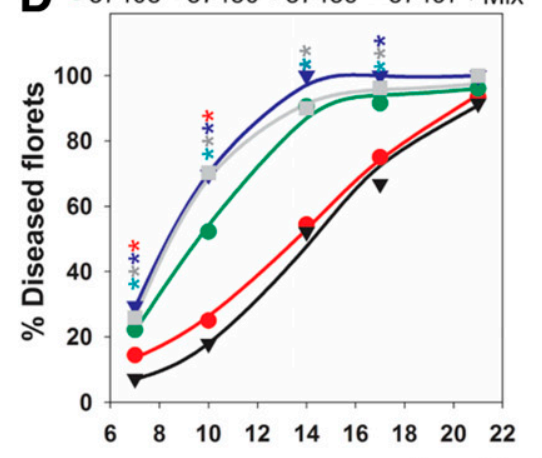

NA2

$\bullet 06-240 \bullet 12 S D 6-2 \nabla 00-588 \backsim 06-239 \nabla \mathrm{Mix}$
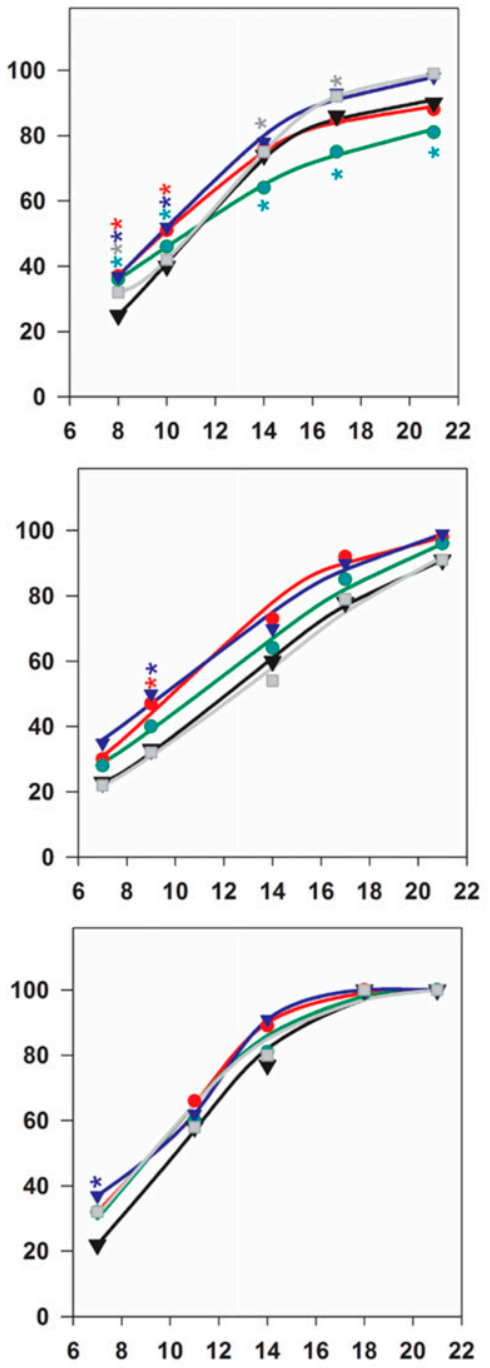

$\bullet 37525 \bullet 52008 \nabla 52195 \square 52429 \nabla \operatorname{Mix}$

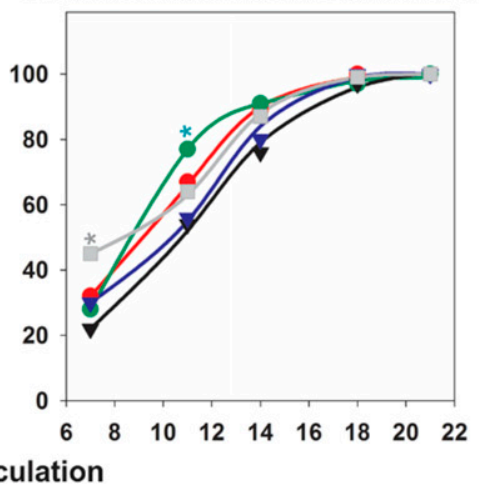

Fig. 2. Intrapopulation interactions influence the rate of disease spread in Norm wheat heads inoculated with NA1 or NA2 Fusarium graminearum. Rates of disease spread (lines) were predicted using regression analysis of average percent diseased florets (filled symbols) for wheat heads inoculated with one of four isolates or a mixture of the four isolates from the NA1 (left) or NA2 (right) population. Asterisks indicate a significantly different percent diseased florets at the given time point for individual isolate inoculations compared with the multi-isolate mix inoculations, based on nonoverlapping, regression predicted confidence intervals. Asterisks are color coded by treatment and placed above the regression curves to indicate significantly higher values or below the curves to indicate significantly lower values. A, B, and C, Results from three replicate experiments using NA1 or NA2 isolates from the Midwestern United States, and D, results from a single experiment using NA1 or NA2 isolates from Canada. Regression equations can be found in Supplementary Table S2. 
number excludes the three significant differences for which 12SD62 isolate had less disease than the NA2 mix. In contrast, 50 significant difference were identified between the disease progression of individual NA1 isolates compared with the NA1 mix. This may indicate that the extent of intrapopulation antagonism was greater for the NA1 population isolates in Norm.

In the moderately resistant cultivar Alsen, multi-isolate mixes similarly displayed reduced disease progression (Fig. 3). Experimental replicates for disease progression were not significantly different $(F$-test, $P>0.05)$, and therefore, were combined for regression analysis. Overall disease progression was less in Alsen in comparison with Norm, and at least three out of the four individual isolates consistently caused significantly more disease than the multi-isolate mixtures for experiments with both U.S. and Canadian isolates. Nevertheless, as was observed in Norm, there were fewer significant differences between single and mixed inoculation for the NA2 population than for NA1. However, this pattern was much less pronounced in Alsen. The number of comparisons showing significantly more disease in heads inoculated with individual isolates compared with the multi-isolate mix was 31 for the NA1 population, and 26 for the NA2 population (Fig. 3A and B). Additionally, the difference between the average percent disease of both U.S. and Canadian NA1 mixes and NA1 individual isolates at day 21 in Alsen was $13 \%$, while the difference between NA2 mixes and NA2 individual isolates was only $5 \%$.

Although most of the observed significant differences indicate that disease progressed faster in heads inoculated with individual isolates, the disease progression rates of the multi-isolate mixtures were generally most similar to the rate of the least aggressive strain in the mixture. This may reflect an inherent intrapopulation competitive advantage for the least aggressive strain during FHB development.

Mycotoxin contamination. The amount of DON contamination in inoculated heads varied across experiments, treatments, and cultivars, therefore the data were analyzed independently for each experiment. Although there were instances in which the amount of DON was significantly different between the individual and multiisolate treatments, there was no obvious trend and the impact of intrapopulation interactions was not clear.

Consistent significant differences between individual isolates and the mix were not observed across replicates for either population in Norm (Fig. 4) or Alsen (Fig. 5). Norm heads inoculated with NA1 isolate $13 \mathrm{MN} 1-6$ had significantly more DON than the NA1 mix in experiment $\mathrm{A}$ and $\mathrm{B}$ but not $\mathrm{C}$ (Fig. 4A to $\mathrm{C}$ ). The amount of DON in Norm inoculated with the individual NA2 isolate 06-239 was also significantly different from the mix in two out of the three experimental replicates, but in experiment $\mathrm{B}$ the amount of DON in heads inoculated with this isolate was less than the NA2 mix (Fig. 4B). In Norm experiment D, the average amount of DON in heads inoculated with the individual Canadian NA2 isolates was consistently higher than the average amount in heads inoculated with the NA2 mix $(P<0.01$; Fig. 4D). In that experiment, the amount of DON recovered from heads inoculated with the NA2 multi-isolate mix was approximately half that recovered from heads inoculated with individual NA2 isolate treatments. In contrast, no significant differences were observed between the Canadian NA1 isolates and the mix. However, a nearly opposite pattern was observed in Alsen, where the amount of DON recovered from heads inoculated with individual Canadian NA2 isolates was not significantly different from the Canadian NA2 mix, but three out of the four Canadian NA1 isolates produced more DON than the NA1 multi-isolate mix $(P<$ 0.01; Fig. 5D).

\section{DISCUSSION}

In this study, we demonstrated that interactions among $F$. graminearum isolates sharing a common population identity and trichothecene chemotype can antagonistically affect the rate of mycelial growth in vitro and FHB development in susceptible and moderately resistant spring wheat (Figs. 1,2, and 3). Coinoculation studies have generally documented competitive interactions among FHB pathogens (Siou et al. 2015a, b; Xu et al. 2007), and intraspecies competition resulting in reduced FHB development has been demonstrated in F. graminearum and F. culmorum (Miedaner et al. 2004; Von der Ohe and Miedaner 2011; Walkowiak et al. 2015). However, this is the first demonstration of intrapopulation competitive interactions among $F$. graminearum.

Our data suggests that the impact of antagonistic intrapopulation interactions may be dependent on the genetic diversity of the population since the observed antagonistic interactions were greater for isolates from the more heterogenous NA1 population. While this difference between the two populations was more pronounced in Norm, a similar trend was also observed in Alsen. The effect of NA2 intrapopulation interaction in Alsen may have become more apparent in the resistant variety because disease progression was substantially reduced, and even the modest intrapopulation interaction between the NA2 isolates became evident. Despite the observed differences in intrapopulation interactions in different cultivars, the observed antagonistic intrapopulation interactions in vitro suggest that these interactions are not dependent on the host or interactions during pathogenicity (Fig. 1).

In a wide variety of microhabitats, the genetic relatedness of microorganisms can influence how they interact (Nadell et al. 2016; Refardt et al. 2013). For example, antagonism has been shown to be stronger among more closely related bacterial species as compared with more phylogenetically distant species, reflecting the evolution of resource competition among microorganisms occupying similar niches (Russel et al. 2017). In filamentous fungi also, interactions between vegetatively incompatible isolates have been shown to impede toxin production more than interactions among compatible isolates (Wicklow and Horn 2007), suggesting that genetic divergence may promote antagonism which is consistent with our findings herein.

Given that competitive behaviors are metabolically costly (West and Gardner 2010), the observed trend of reduced rate of FHB progression in wheat heads inoculated with mixtures of multiple isolates may reflect a functional trade-off, where for example, metabolic functions of the pathogen shift from pathogenicity to competition when a conspecific isolate is encountered within the host. Accordingly, mixed infections are often less virulent than single strain infections (Garbutt et al. 2011). Nevertheless, results herein argue that population background and diversity may also be influencing pathogen interactions and FHB spread in planta. In other filamentous fungi, more genetically divergent isolates are more likely than closely related ones to compete in planta (Koskella et al. 2006). As such, we would expect higher competition among NA1 isolates within the host because they are more genetically diverse than NA2 isolates. Additionally, populations may have different defense strategies, which is supported by extensive divergence of genes involved in fungal immunity and self-defense observed between NA1 and NA2 F. graminearum (Kelly and Ward 2018). Accordingly, there are examples in the literature of microbial toxins that are lethal to nonrelatives, but harmless to closely related isolates that possess a genetically linked immunity gene (Riley and Wertz 2002). Although it is not known if NA1 or NA2 isolates secrete such toxins in planta, molecular and metabolic evaluations would likely clarify defense strategies of different $F$. graminearum populations throughout the infection process.

It is possible that the observed differences between the two populations are not due to differences in genetic diversity but rather due to differences in population aggressiveness. At least in Alsen, the rate of disease progression in heads inoculated with the mixture was generally similar to that of the least aggressive individual strain in the mixture. Although we are unaware of examples of the least aggressive strain being more competitive, our data cannot exclude the possibility that the least aggressive strains had some inherent 
characteristics that allowed them to more successfully establish and exclude the other strains from the rachis. Further research is needed to confirm or exclude this possibility.

In contrast to interactions between $F$. graminearum isolates of different chemotypes (Walkowiak et al. 2015), DON production was not consistently reduced by intrapopulation interactions among isolates with the same chemotype, nor was there any consistent trend
(Figs. 4 and 5). The effect may be dependent on the interacting strains. F. graminearum isolates infecting the same wheat head may rely on interference competition which typically involves some form of behavioral or chemical interaction between individuals prior to actual use of common resources (Wicklow 1981). However, since the competing isolates in these experiments also had the same chemotype, it is unlikely that trichothecenes would be involved
A

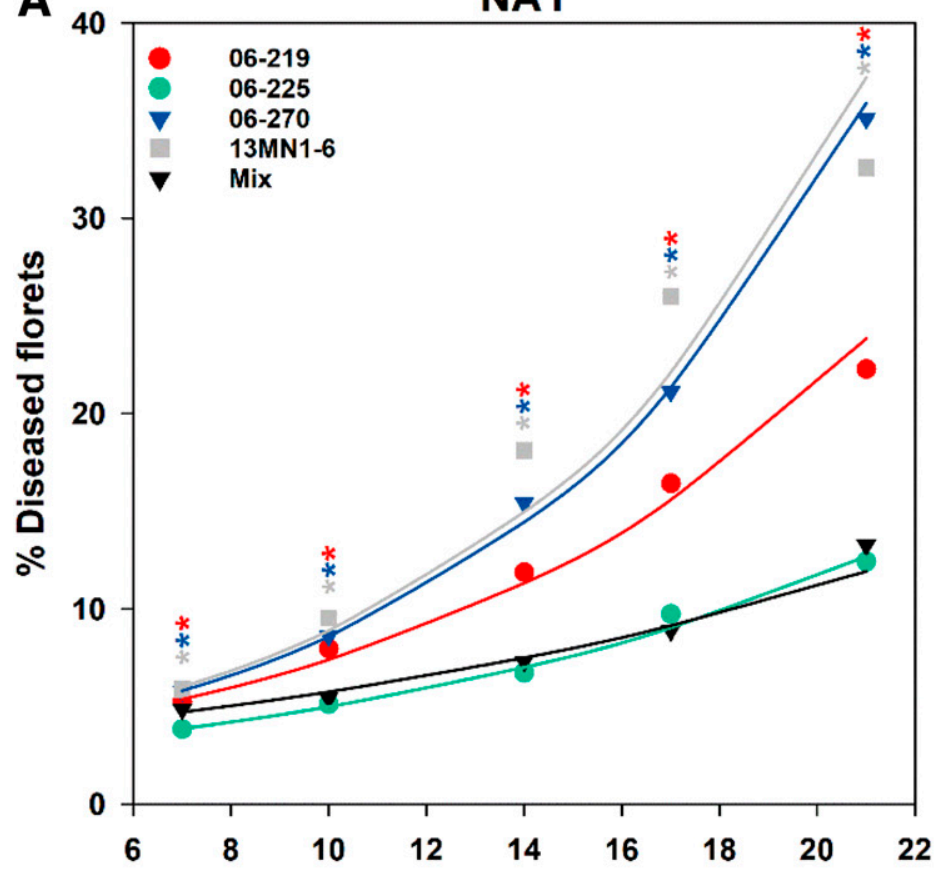

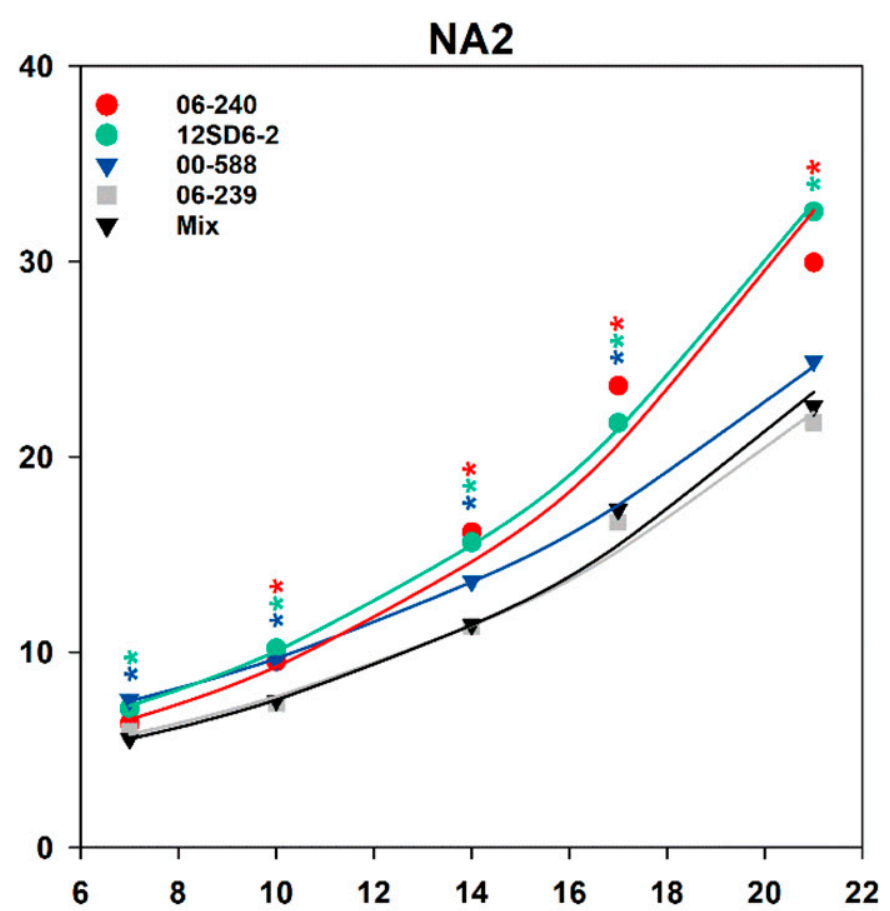

B

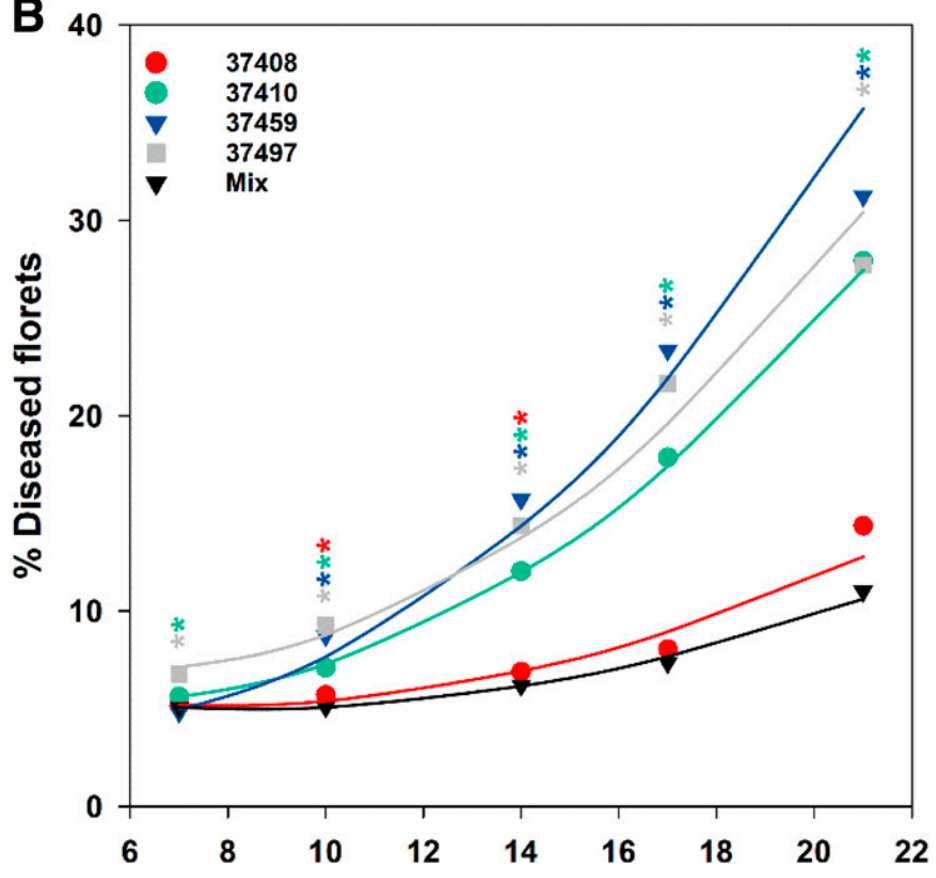

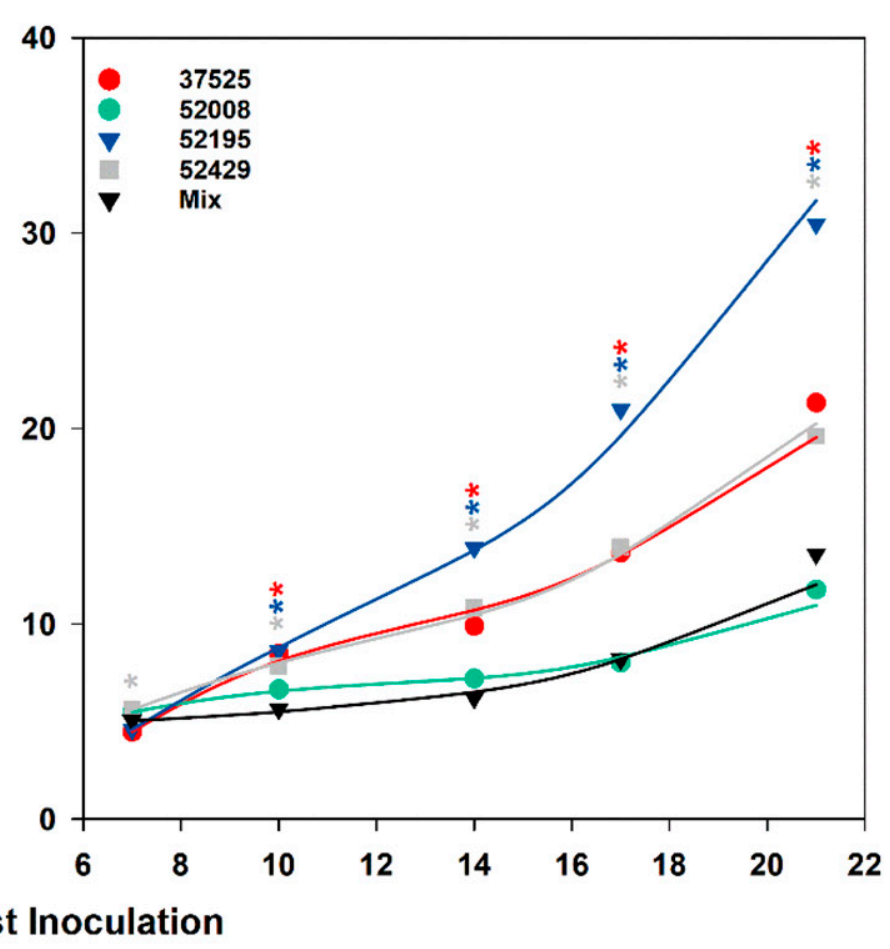

40

2

Days Post Inoculation

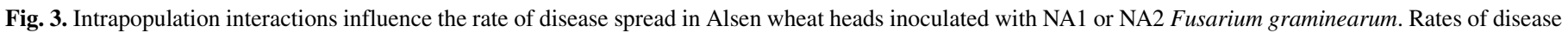

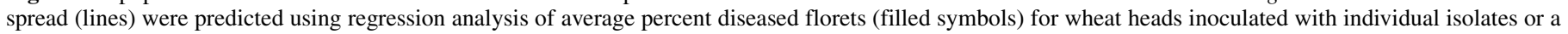

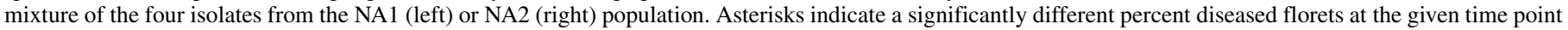

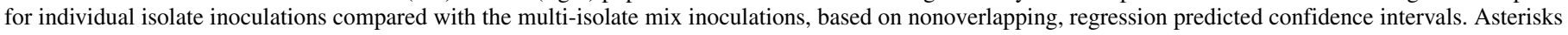

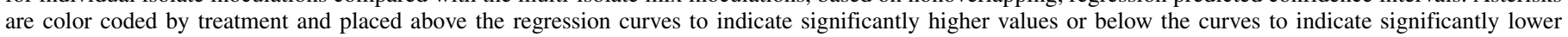

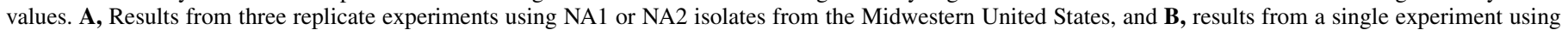
NA1 or NA2 isolates from Canada. Regression equations can be found in Supplementary Table S3. 
their competitive interactions. Additionally, greenhouse experiments that measured toxin content of the entire wheat head, as herein, have shown that interspecies competition between FHB pathogens does not consistently affect toxin production, but occasionally, isolate-specific changes in toxin production were observed on some coinoculated heads (Siou et al. 2015b). It is also important to consider that the toxin analyses were performed at 21 DPI. Differences in DON content caused by pathogen interactions may not be detectable at the later stages of infection because competition between different FHB pathogens can result in competitive exclusion on a single head (Siou et al. 2015a). Therefore, DON analyses of multi-isolate inoculated heads may reflect the cumulative toxin
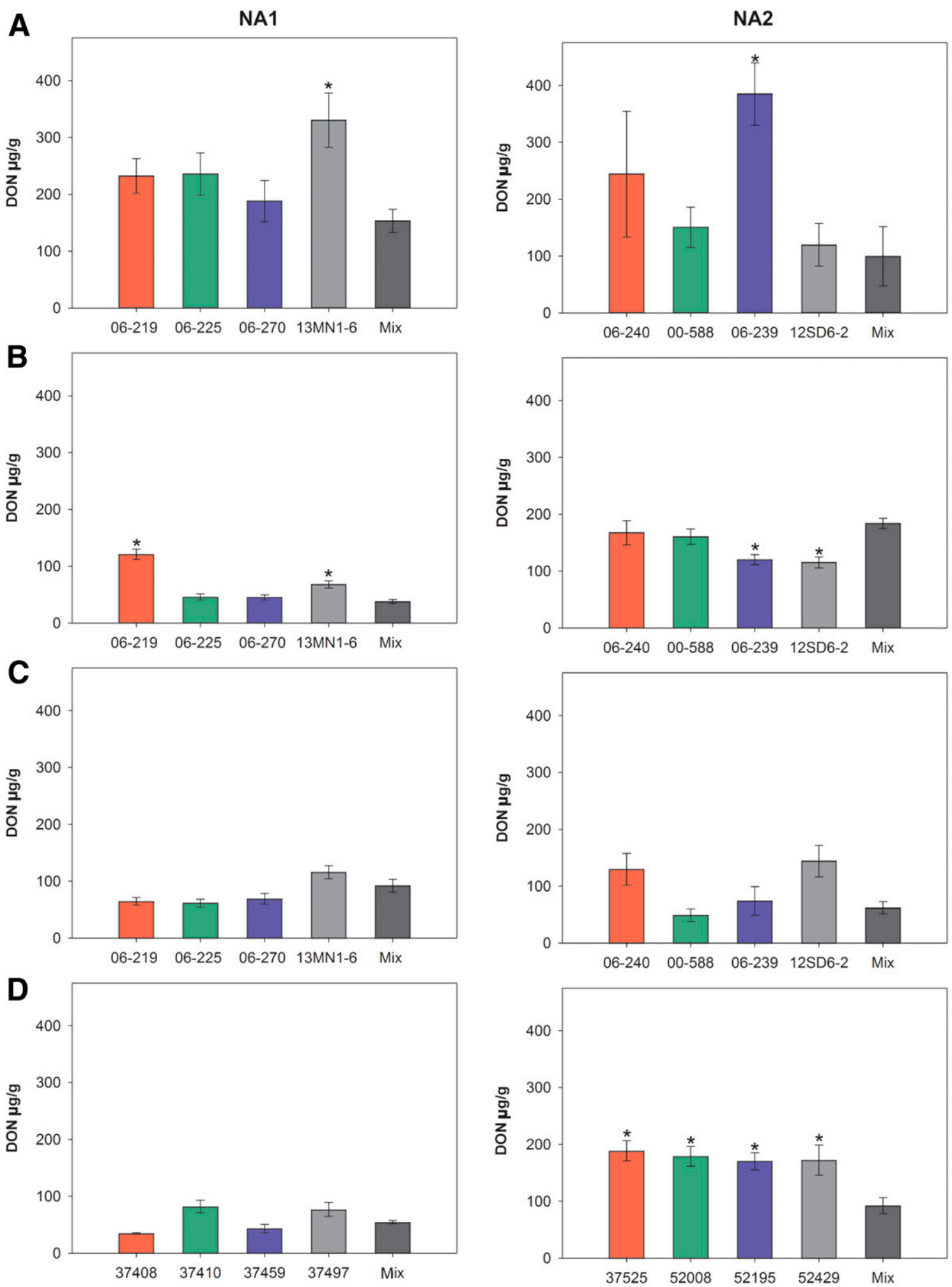

Fig. 4. Deoxynivalenol (DON) concentration (micrograms per gram of dry plant tissue) in Norm wheat heads inoculated with individual isolates compared with multi-isolate mixtures. Average DON concentration \pm standard error in Norm wheat heads 21 days postinoculation with individual isolates or a mixture of the four isolates from the corresponding NA1 (left) or NA2 (right) populations. A, B, and C, Data from the set of Midwestern U.S. Fusarium graminearum isolates, and D, data from the set of Canadian isolates. Asterisks denote individual isolate treatments that produced significantly different amounts of DON compared with the multi-isolate mix (Dunnett's or Steel's test, $P<0.05$ ). 
production of the most competitive isolate(s), which may not always be the most aggressive or highest toxin producer (Von der Ohe and Miedaner 2011). As such, further research investigating the FHB dynamics of single isolate infections versus multiple isolate infections is warranted.

Previous studies have documented the frequent co-occurrence of genetically distinct isolates of $F$. graminearum and $F$. culmorum
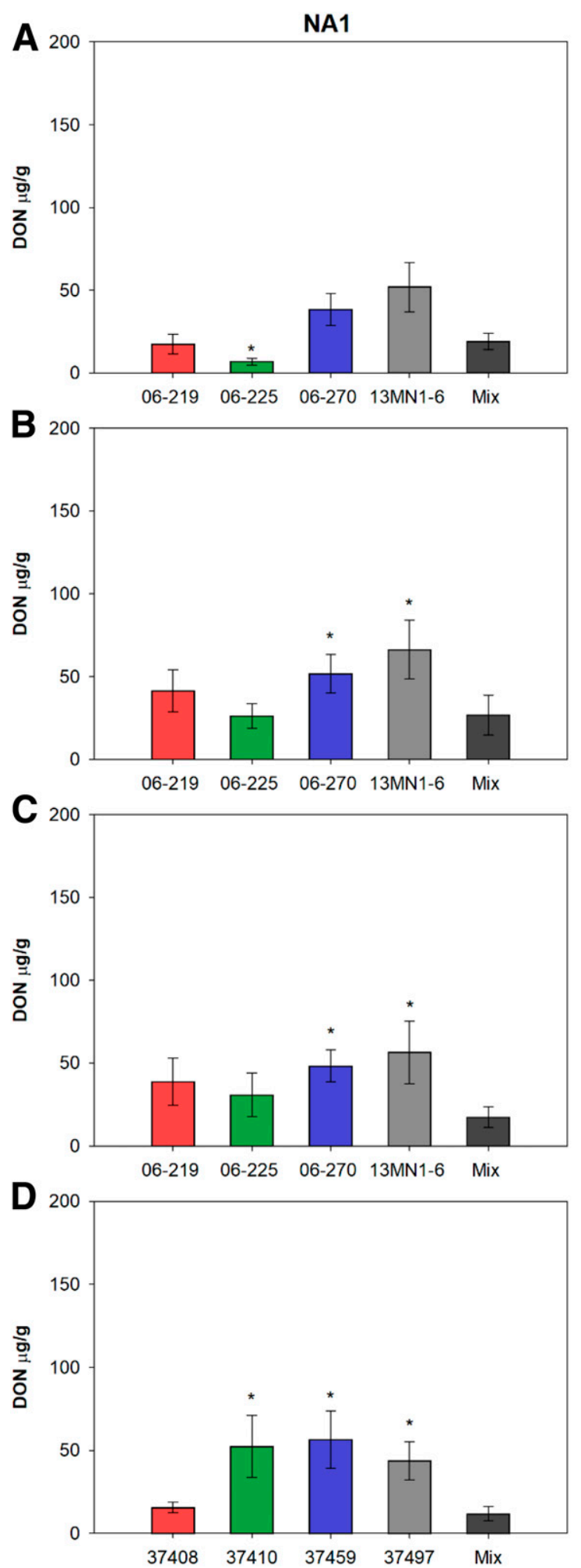

within the same wheat spike (Miedaner et al. 2001, 2004). In some areas, a single $F$. graminearum genetic population and chemotype dominates the fields (Bec et al. 2015; Gale et al. 2011; Talas and McDonald 2015), but in others there have been reports of $F$. graminearum isolates with different chemotypes and genetic backgrounds being sampled from the same host plant (Kelly et al. 2015; McCallum et al. 2001).
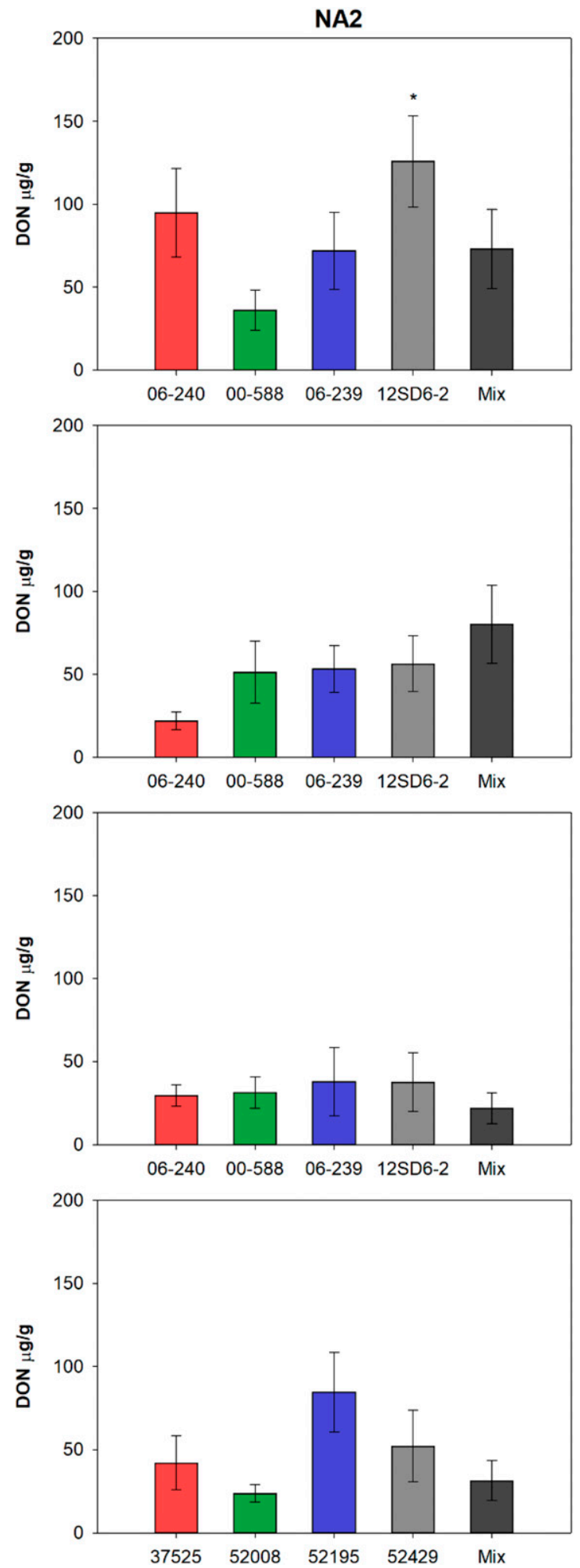

Fig. 5. Deoxynivalenol (DON) concentration (micrograms per gram of dry plant tissue) in Alsen wheat heads inoculated with individual isolates compared with multi-isolate mixtures. Average DON concentration \pm standard error in Alsen wheat heads 21 days postinoculation with individual isolates or a mixture of the four isolates from the corresponding NA1 (left) or NA2 (right) populations. A, B, and C, Data from the set of Midwestern U.S. Fusarium graminearum isolates, and D, data from the set of Canadian isolates. Asterisks denote individual isolate treatments that produced significantly different amounts of DON compared with the multi-isolate mix (Dunnett's or Steel's test, $P<0.05$ ). 
Given that $F$. graminearum isolates can coinfect the same host plant under natural field conditions (Kelly et al. 2015; McCallum et al. 2001), knowledge of potential antagonistic intraspecies interaction could benefit FHB management and research. If intrapopulation antagonism occurs in the field similarly to the demonstration herein, knowledge of local pathogen populations could provide additional information regarding the window of opportunity to apply fungicide and reduce disease spread, or best practices for cultivar selection. For example, susceptible wheat varieties in fields harboring diverse NA1 $F$. graminearum isolates will likely experience a lower rate of FHB development, increasing the window of opportunity to apply fungicide, while fields dominated by NA2 isolates may experience more aggressive FHB progression and reduced fungicide efficacy if applied late. On the other hand, fields with moderately resistant wheat varieties will likely benefit from antagonistic intrapopulation interactions regardless of pathogen population.

From a management perspective, the rate of disease progression may impact the ability of a farmer to effectively react. Recent studies that have suggested that fungicide treatments applied after anthesis can still reduce FHB spread (D'Angelo et al. 2014; Freije and Wise 2015; Wegulo et al. 2013). However, these studies and many others that have evaluated disease spread and the efficacy of resistant cultivars and fungicides have attempted to represent native field inoculum by performing artificial inoculations with a mixture of endemic isolates (D'Angelo et al. 2014; Foroud et al. 2012; Freije and Wise 2015; Salgado et al. 2011). Our data indicate that wheat heads inoculated with multiple isolates generally display disease spread similar to the least aggressive strain (Figs. 2 and 3) and not an average of the disease progression rate of the isolates within the mix. Therefore, disease assays using isolate coinoculation methods may underestimate disease conditions that can be caused by the more aggressive isolates.

\section{ACKNOWLEDGMENTS}

We thank Amy Kelly whose contributions made this manuscript possible; the anonymous reviewers whose suggestions improved the description and interpretation of the results in this manuscript; and our exceptional technical staff: Jennifer Teresi, Thomas Usgaard, Stephanie Folmar, and Christine J. Hodges.

\section{LITERATURE CITED}

Bai, G.-H., Desjardins, A., and Plattner, R. 2002. Deoxynivalenol-nonproducing Fusarium graminearum causes initial infection, but does not cause disease spread in wheat spikes. Mycopathologia 153:91-98.

Baldwin, T., Baldwin, S., Klos, K., Bregitzer, P., and Marshall, J. 2019. Deletion of the benzoxazinoid detoxification gene NAT1 in Fusarium graminearum reduces deoxynivalenol in spring wheat. PLoS One 14:e0214230.

Bec, S., Ward, T., Farman, M., O’Donnell, K., Hershman, D., Van Sanford, D., and Vaillancourt, L. J. 2015. Characterization of Fusarium strains recovered from wheat with symptoms of head blight in Kentucky. Plant Dis. 99: $1622-1632$.

D’Angelo, D., Bradley, C., Ames, K., Willyerd, K., Madden, L., and Paul, P. 2014. Efficacy of fungicide applications during and after anthesis against Fusarium head blight and deoxynivalenol in soft red winter wheat. Plant Dis. 98:1387-1397.

Dunnett, C. W. 1955. A multiple comparison procedure for comparing several treatments with a control. J. Am. Stat. Assoc. 50:1096-1121.

Foroud, N., McCormick, S., MacMillan, T., Badea, A., Kendra, D., Ellis, B., and Eudes, F. 2012. Greenhouse studies reveal increased aggressiveness of emergent Canadian Fusarium graminearum chemotypes in wheat. Plant Dis. 96:1271-1279.

Freije, A. N., and Wise, K. A. 2015. Impact of Fusarium graminearum inoculum availability and fungicide application timing on Fusarium head blight in wheat. Crop Prot. 77:139-147.

Gale, L. R., Harrison, S. A., Ward, T. J., O'Donnell, K., Milus, E. A., Gale, S. W., and Kistler, H. C. 2011. Nivalenol-type populations of Fusarium graminearum and $F$. asiaticum are prevalent on wheat in southern Louisiana. Phytopathology 101:124-134.

Gale, L. R., Ward, T. J., Balmas, V., and Kistler, H. C. 2007. Population subdivision of Fusarium graminearum sensu stricto in the Upper Midwestern United States. Phytopathology 97:1434-1439.
Garbutt, J., Bonsall, M. B., Wright, D. J., and Raymond, B. 2011. Antagonistic competition moderates virulence in Bacillus thuringiensis. Ecol. Lett. 14: 765-772.

Gilbert, J., Clear, R. M., Ward, T. J., Gaba, D., Tekauz, A., Turkington, T. K., Woods, S. M., Nowicki, T., and O'Donnell, K. 2010. Relative aggressiveness and production of 3- or 15-acetyl deoxynivalenol and deoxynivalenol by Fusarium graminearum in spring wheat. Can. J. Plant Pathol. 32: 146-152.

Goswami, R. S., and Kistler, H. C. 2004. Heading for disaster: Fusarium graminearum on cereal crops. Mol. Plant Pathol. 5:515-525.

Kelly, A. C., Clear, R. M., O’Donnell, K., McCormick, S., Turkington, K., Tekauz, A., Gilbert, J., Kistler, H. C., Busman, M., and Ward, T. J. 2015. Diversity of Fusarium head blight populations and trichothecene toxin types reveals regional differences in pathogen composition and temporal dynamics. Fungal Genet. Biol. 82:22-31.

Kelly, A. C., and Ward, T. J. 2018. Population genomics of Fusarium graminearum reveals signatures of divergent evolution within a major cereal pathogen. PLoS One 13:e194616.

Koskella, B., Giraud, T., and Hood, M. 2006. Pathogen relatedness affects the prevalence of within-host competition. Am. Nat. 168:121-126.

Liang, J., Lofgren, L., Ma, Z., Ward, T. J., and Kistler, H. C. 2015. Population subdivision of Fusarium graminearum from barley and wheat in the Upper Midwestern United States at the turn of the century. Phytopathology 105: 1466-1474.

McCallum, B., Tekauz, A., and Gilbert, J. 2001. Vegetative compatibility among Fusarium graminearum (Gibberella zeae) isolates from barley spikes in southern Manitoba. Can. J. Plant Pathol. 23:83-87.

McMullen, M., Bergstrom, G., De Wolf, E., Dill-Macky, R., Hershman, D., Shaner, G., and Van Sanford, D. 2012. A unified effort to fight an enemy of wheat and barley: Fusarium head blight. Plant Dis. 96:1712-1728.

Mesterhazy, A., Bartok, T., Mirocha, C., and Komoroczy, R. 1999. Nature of wheat resistance to Fusarium head blight and the role of deoxynivalenol for breeding. Plant Breed. 118:97-110.

Miedaner, T., Schilling, A. G., and Geiger. 2004. Competition effects among isolates of Fusarium culmorum differing in aggressiveness and mycotoxin production on heads of winter rye. Eur. J. Plant Pathol. 110:63-70.

Miedaner, T., Schilling, A. G., and Geiger, H. H. 2001. Molecular genetic diversity and variation for aggressiveness in populations of Fusarium graminearum and Fusarium culmorum sampled from wheat fields in different countries. J. Phytopathol. 149:641-648

Miller, J. D., Greenhalgh, R., Wang, Y., and Lu, M. 1991. Trichothecene chemotypes of three Fusarium species. Mycologia 83:121-130.

Mogg, C., Bonner, C., Wang, L., Schernthaner, J., Smith, M., Desveaux, D., and Subramaniam, R. 2019. Genomic identification of the tor signaling pathway as a target of the plant alkaloid antofine in the phytopathogen Fusarium graminearum. MBio 10:e00792-19.

Nadell, C. D., Drescher, K., and Foster, K. R. 2016. Spatial structure, cooperation and competition in biofilms. Nat. Rev. Microbiol. 14:589-600.

Nganje, W. E., Kaitibie, S., Wilson, W. W., Leistritz, F. L., and Bangsund, D. A. 2004. Economic impacts of Fusarium head blight in wheat and barley. Pages 1993-2001 in: Agribusiness and Applied Economics Report No. 538, vol. 2014. Department of Agribusiness and Applied Economics, North Dakota State University, ND.

Pestka, J. 2010. Toxicological mechanisms and potential health effects of deoxynivalenol and nivalenol. World Mycotoxin J. 3:323-347.

Puri, K. D., and Zhong, S. 2010. The 3ADON population of Fusarium graminearum found in North Dakota is more aggressive and produces a higher level of DON than the prevalent 15ADON population in spring wheat. Phytopathology 100:1007-1014.

Refardt, D., Bergmiller, T., and Kümmerli, R. 2013. Altruism can evolve when relatedness is low: Evidence from bacteria committing suicide upon phage infection. Proc. R. Soc. Lond. B Biol. Sci. 280:20123035.

Riley, M. A., and Wertz, J. E. 2002. Bacteriocins: Evolution, ecology, and application. Annu. Rev. Microbiol. 56:117-137.

Russel, J., Røder, H. L., Madsen, J. S., Burmølle, M., and Sørensen, S. J. 2017. Antagonism correlates with metabolic similarity in diverse bacteria. Proc. Natl. Acad. Sci. 114:10684-10688.

Salgado, J. D., Wallhead, M., Madden, L. V., and Paul, P. A. 2011. Grain harvesting strategies to minimize grain quality losses due to Fusarium head blight in wheat. Plant Dis. 95:1448-1457.

Siou, D., Gélisse, S., Laval, V., Elbelt, S., Repinçay, C., Bourdat-Deschamps, M., Suffert, F., and Lannou, C. 2015b. Interactions between head blight pathogens: Consequences for disease development and toxin production in wheat spikes. Appl. Environ. Microbiol. 81:957-965.

Siou, D., Gélisse, S., Laval, V., Suffert, F., and Lannou, C. 2015a. Mutual exclusion between fungal species of the Fusarium head blight complex in a wheat spike. Appl. Environ. Microbiol. 81:4682-4689.

Steel, R. G. 1959. A multiple comparison rank sum test: Treatments versus control. Biometrics 15:560-572. 
Talas, F. and McDonald, B. A. 2015. Genome-wide analysis of Fusarium graminearum field populations reveals hotspots of recombination. BMC Genomics 16:996.

Von der Ohe, C., and Miedaner, T. 2011. Competitive aggressiveness in binary mixtures of Fusarium graminearum and $F$. culmorum isolates inoculated on spring wheat with highly effective resistance QTL. J. Phytopathol. 159: 401-410.

Wagacha, J. M., Oerke, E. C., Dehne, H. W., and Steiner, U. 2012. Interactions of Fusarium species during prepenetration development. Fungal Biol. 116: 836-847.

Walkowiak, S., Bonner, C. T., Wang, L., Blackwell, B., Rowland, O., and Subramaniam, R. 2015. Intraspecies interaction of Fusarium graminearum contributes to reduced toxin production and virulence. Mol. Plant-Microbe Interact. 28:1256-1267.

Ward, T. J., Clear, R. M., Rooney, A. P., O’Donnell, K., Gaba, D., Patrick, S., Starkey, D. E., Gilbert, J., Geiser, D. M., and Nowicki, T. W. 2008. An adaptive evolutionary shift in Fusarium head blight pathogen populations is driving the rapid spread of more toxigenic Fusarium graminearum in North America. Fungal Genet. Biol. 45:473-484.

Wegulo, S. N., Bockus, W. W., Nopsa, J. F. H., Peiris, K. H., and Dowell, F. E. 2013. Integration of fungicide application and cultivar resistance to manage Fusarium head blight in wheat. Chapter 2 in: Fungicides-Showcases of Integrated Plant Disease Management from Around the World. InTechOpen. doi:10.5772/3251.

Welch, B. L. 1947. The generalization of 'student's' problem when several different population variances are involved. Biometrika 34:28-35.

West, S. A., and Gardner, A. 2010. Altruism, spite, and greenbeards. Science 327:1341-1344

Wicklow, D. T. 1981. Interference competition and the organization of fungal communities. Pages 351-375 in: The Fungal Community. D. Wicklow and G. Corrol, eds. Marcel Dekker, New York.

Wicklow, D. T., and Horn, B. W. 2007. Association between vegetative compatibility and aflatoxin production by Aspergillus species during intraspecific competition. Mycoscience 48:267-273.

Wu, F., Groopman, J. D., and Pestka, J. J. 2014. Public health impacts of foodborne mycotoxins. Annu. Rev. Food Sci. Technol. 5:351-372.

Xu, X. M., Monger, W., Ritieni, A., and Nicholson, P. 2007. Effect of temperature and duration of wetness during initial infection periods on disease development, fungal biomass and mycotoxin concentrations on wheat inoculated with single, or combinations of Fusarium species. Plant Pathol. 56:943-956.

Xu, X.-M., Parry, D., Nicholson, P., Thomsett, M., Simpson, D., Edwards, S., Cooke, B., Doohan, F., Brennan, J., and Moretti, A. 2005. Predominance and association of pathogenic fungi causing Fusarium ear blight in wheat in four European countries. Eur. J. Plant Pathol. 112:143-154. 